\title{
Implication of TYMP genetic variation on the prognosis of patients with colorectal cancer who received capecitabine-based adjuvant chemotherapy after surgical resection: a real-world exploratory study
}

Xinyu Peng ( $\nabla$ pengxinyu2010@163.com )

affiliated hospital of Hebei University https://orcid.org/0000-0002-2692-9526

Tao Zhang affiliated hospital of hebei university

Junjie Sun

affiliated hospital of hebei university

Hengxue Lin

affiliated hospital of hebei university

Tianliang Bai

affiliated hospital of hebei university

Yating Qiao

affiliated hospital of hebei university

Yaxin Li

affiliated hospital of hebei university

Gang Li

affiliated hospital of hebei university

Guicun Li

affiliated hospital of hebei university

Xiongjie Jia

affiliated hospital of hebei university

Aimin Zhang

affiliated hospital of hebei university

\section{Research Article}

Keywords: colorectal cancer, adjuvant chemotherapy, capecitabine, prognosis, polymorphism

Posted Date: October 25th, 2021 
DOl: https://doi.org/10.21203/rs.3.rs-966707/v1

License: (c) (1) This work is licensed under a Creative Commons Attribution 4.0 International License. Read Full License 


\section{Abstract \\ Background}

Thymidine Phosphorylase (TYMP) gene was of crucial significance in the process of colorectal cancer (CRC) development and also played an important role in capecitabine metabolism. Present study was to identify the association between TYMP polymorphism and prognosis of postoperative patients with CRC who received capecitabine-based adjuvant chemotherapy.

\section{Methods}

A total of 218 patients with CRC who were treated with surgical resection and capecitabine-based adjuvant chemotherapy were included in this study retrospectively. Peripheral blood and peripheral blood mononuclear cell (PBMC) specimen of the patients with CRC were collected for the genotyping of TYMP polymorphism and TYMP mRNA expression, respectively. Univariate analysis of genotypes and prognosis was carried out by Kaplan-Meier survival analysis, and multivariate were adjusted by Cox regression analysis. Expression of TYMP according to genotype status was analyzed using non-parameter test.

\section{Results}

The median disease-free survival (DFS) of the 218 patients with CRC was 4.6 years, and the median overall survival (OS) was 5.8 years. Regarding the polymorphism analysis, only rs 11479 was of clinical significance. The prevalence of rs 11479 in TYMP among the 218 patients indicated that minor allele frequency was 0.20 (GG 141 cases, GA 68 cases and AA 9 cases), which accorded with Hardy-Weinberg Equilibrium ( $P=0.825)$. Prognostic analysis according to rs11479 genotype status suggested that the median DFS of patients with GG genotype and GA/AA genotype was 3.1 and 6.1 years, respectively $(P=0.004)$. Furthermore, the median OS of patients with $\mathrm{GG}$ genotype and $\mathrm{GA} / \mathrm{AA}$ genotype was 5.0 and 7.0 years, respectively $(P=0.033)$. In order to adjust the confounding factors which might contribute to OS, a multivariate Cox regression analysis was introduced and the results exhibited that rs11479 polymorphism was an independent factor for DFS (HR=1.64, $P=0.009)$. Additionally, of the 65 PBMC specimens, the mRNA expression results indicated that patients with GA/AA genotypes conferred significantly higher mRNA expression of TYMY than that of patients with GG genotype $(P<0.001)$.

\section{Conclusions}

TYMP gene polymorphism rs11479 might involve in the prognosis of patients with CRC who received capecitabine based adjuvant chemotherapy through mediation of the mRNA expression of TYMP. Conclusion of present study should be confirmed in prospective clinical trial subsequently. 


\section{Background}

Colorectal cancer (CRC) is one of the most common gastrointestinal tumors worldwide. It is estimated that there are approximately 56,000 new cases and 29,000 new deaths of CRC in China currently according to the recent epidemiological data[1]. To our knowledge, surgical resection was established to be the only way to cure patients with CRC for decades[2]. Unfortunately, approximately $30 \%$ of the patients were diagnosed of distant metastases, thus missing the opportunity for surgical resection[3]. Consequently, almost half of the patients with CRC were able to receive surgical resection clinically. Recent years had witnessed in-depth development of molecular typing in the field of colon cancer or rectal cancer with advanced stage, more and more targeted drugs with different targets of action were proved to provide considerable treatment options, thus bringing more survival benefits for the patients consecutively[4]. Nevertheless, the meaningful research progress in early-stage and postoperative patients with CRC treatment was still limited comparatively. Only IDEA trial was proved to be the significant breakthrough with clinical significance recently, which suggested that 3 months rather than 6 months of adjuvant chemotherapy was recommended for low-risk stage $\Downarrow$ colon cancer receiving capecitabine plus oxaliplatin regimen[5]. Conclusions in IDEA trial helped to attenuate the unnecessary side reactions of chemotherapy to some extent in view of the fact that 6 months adjuvant chemotherapy regimens had been established to be the standard of care for stage $₫$ patients with CRC for decades[6]. Besides, with the adequate development of the more sensitive detection techniques, ctDNA had established to be the potential predictive value in the recurrence risk assessment for patients with CRC after surgical resection[7]. However, the predictive role regarding ctDNA for the intensity or duration of adjuvant chemotherapy among patients with CRC was still controversial and no standardized and unified criteria was available for the corresponding detection of ctDNA currently[8].

Capecitabine-based adjuvant chemotherapy had been established as the standard of care for the patients with CRC after surgical resection for decades, which improved 5-year survival rates of approximately $10 \%$ [9]. However, the benefits of capecitabine-based adjuvant chemotherapy for patients with stage $\triangle \mathrm{CRC}$ regarding reducing recurrence and prolonging survival remained controversial currently[10]. Furthermore, large individual differences were still observed in the clinical application of capecitabine-based adjuvant chemotherapy, which suggested that many factors might compromise the prognosis of this regimen clinically. Capecitabine can be transformed into 5-FU only through the key metabolism enzyme of thymidine phosphorylase (TYMP), thus playing the cytotoxic effects to kill the tumor[11]. Furthermore, previous study indicated that TYMP gene was similar with the structure of platelet-derived endothelial cell growth factor in tumor cells, which played an important role in promoting tumor angiogenesis and metastasis[12]. Therefore, many studies had confirmed that TYMP was highly expressed in various kinds of tumors.

TYMP gene was located at chromosome $22 \mathrm{q} 13.33$ and contained 10 exons. As a member of the endothelial vascular growth factor family, TYMP gene was not conserved in Chinese population, which suggested that expression of TYMP also showed great individual differences among different individuals[13]. Relatively limited studies regarding the polymorphism study of this gene in Chinese 
population was observed. Especially in the field of colorectal cancer, rare research had been performed on this gene polymorphism currently[14]. Previous work initiated by YB Du and colleagues implemented the exploration of TYMP genetic variation on clinical outcome and safety of CRC[15]. And they identified that polymorphism of TYMP could be of potential significance clinically. Besides, another study initiated by L Huang and colleagues investigated the clinical significance of TYMP gene polymorphism among patients with advanced gastrointestinal tumors[16]. Conclusion exhibited that the expression level of TYMP gene was higher among patients with T allele in the advanced gastrointestinal tumors.

Nevertheless, the relevance of TYMP polymorphism to the prognosis of real-world patients with CRC who received capecitabine based adjuvant chemotherapy after surgical resection remained unknown.

Consequently, present study aimed to identify the association between TYMP polymorphism and prognosis of patients with CRC who received surgical resection and capecitabine-based adjuvant chemotherapy in real-world.

\section{Methods}

\section{Patients}

Given that capecitabine-based adjuvant chemotherapy was licensed in Chin over ten years, and considerable patients with CRC had received capecitabine-based adjuvant chemotherapy clinically. Therefore, present study was designed as real-world retrospective research. Appropriate patients with CRC underwent surgical resection were collected in the department of Gastrointestinal surgery of Affiliated Hospital of Hebei University from January 2010 to March 2021. Baseline characteristics of the patients were obtained from the hospitals electronic medical record system. Patients who fulfilled the eligibility criteria were included in the study retrospectively. Inclusion criteria manifested as: (1) aged $\geq 18$ years; (2) ECOG performance status of 0-2 score; (3) adequate cardiac function, renal function and bone marrow function to receive adjuvant chemotherapy appropriately; (4) pathological diagnosis of colon cancer or rectal cancer; (5) received surgical resection and postoperative adjuvant chemotherapy; (6) pathological

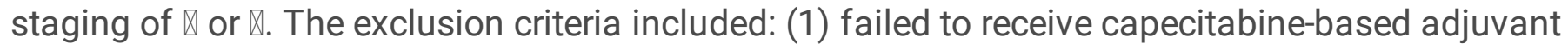
chemotherapy (capecitabine monotherapy or capecitabine related regimens) after surgical resection; (2) peripheral blood specimen was not available for DNA extraction; (3) diagnosed of familial adenomatous polyposis or hereditary CRC; (4) concomitant with another tumor or serious diseases that might compromise the survival of the patients. Flow chart of present study was illustrated in Figure 1. Finally, a total of 218 patients with CRC patients met the eligibility criteria were enrolled in this study. The primary endpoint of present study was the association between prognosis and polymorphism genotype status. The study was approved by the ethics committee of the Affiliated Hospital of Hebei University. Informed consent was signed by each enrolled patient in accordance with the recommendation of the declaration of Helsinki.

\section{Adjuvant Chemotherapy Regimens And Follow Up Protocol}


Adjuvant chemotherapy regimens and follow up protocol

All the patients with CRC included in this study were treated with capecitabine-based adjuvant chemotherapy. The chemotherapy regimens included capecitabine monotherapy and CAPEOX combination therapy. And the usage and dosage of capecitabine monotherapy was as follows: 3-4 weeks after surgical treatment, capecitabine, $1000-1250 \mathrm{mg} / \mathrm{m}^{2}$, twice daily, day 1-14, every 21 days as one cycle. Additionally, the usage and dosage of CAPEOX regimen manifested as: capecitabine $1000 \mathrm{mg} / \mathrm{m}^{2}$, twice daily, day $1-14$, every 21 days as one cycle, combined with oxaliplatin, $80-130 \mathrm{mg} / \mathrm{m}^{2}$, iv. infusion, day 1 . The adjuvant chemotherapy period was planned for 8 cycles or depended on the actual situation of the patients.

Each patient was followed up at the onset of adjuvant chemotherapy administration. Initial follow-up was implemented when the patient received adjuvant chemotherapy in the hospital, where the baseline characteristics and the date of disease recurrence could be obtained through the electronic medical record system specifically. Subsequent follow-up was performed mainly by telephone. Patients were followed up every three months for the date of recurrence and treatment after recurrence, and the death status were mainly inquired. Furthermore, when a patient was recurrence or death, it must be confirmed by at least two research colleagues before it could be confirmed as a recurrence events or death events.

\section{Collection Of Peripheral Blood Specimens And Tymp Polymorphism Analysis}

Peripheral blood specimen from each enrolled patient were collected when it was available. Genomic DNA was extracted using the traditional phenol chloroform method. Unfortunately, 19 patients failed to obtain the available peripheral blood specimen and 12 patients failed for the genomic DNA extraction. Consequently, a total of 218 patients with adjuvant chemotherapy had the suitable DNA specimens and included in the polymorphism analysis finally. The single nucleotide polymorphism included in this study were collected from the NCBI database with the minor allele frequency $>10 \%$ among Chinese population or the previous study that identified the clinical significance of the polymorphisms. Consequently, polymorphisms included were rs11479, rs131804 and rs470119. In the preliminary analysis between the polymorphism status and prognosis, only rs11479 was significantly associated with prognosis. As a result, the subsequent analysis of present study was focused on rs11479 polymorphism accordingly.

The rs11479 polymorphism was genotyped using the method of polymerase chain reaction-restriction fragment length polymorphism (PCR-RFLP). PCR product of rs11479 was amplified initially, the PCR product including this polymorphism was amplified, forward primer was 5'- TCTAACAGCCCCTCGCTCT-3', reverse primer was 5'- GGGTCACGTGTTCATCGAG-3'. Size of PCR product was 266bp. And a total of $2 \mu \mathrm{L}$ PCR products were digested using the restriction enzyme Ndel (Thermo Fisher Scientific, USA). Genotype status of rs11479 were distinguished through the size of PCR bands according to the previous study[17]. Additionally, Genotyping results for rs11479 were confirmed in some randomly selected samples using 
ionization-time-of-flight mass spectrometry (Sequenom, SanDiego, CA) method. And the two genotyping approaches reached a $100 \%$ consistency.

\section{Peripheral blood Mononuclear Cell (PBMC) specimen collection and TYMP gene mRNA expression analysis}

As described in the flow chart of present study, PBMC specimens were collected in the 77 randomly selected subjects from the 218 patients with CRC initially. However, 7 patients' PBMC specimens were not available and 5 patients failed for RNA extraction experiment subsequently. Eventually, a total of 65 mRNA specimens were available for the subsequent analysis and preserved in Liquid nitrogen. Total RNA samples were extracted using TRizol reagents (Takara Biotechnology, China) according to the manufacture's instruction and stored at $-80^{\circ} \mathrm{C}$ for mRNA expression analysis. A total of 500ng RNA extracted from the PBMC specimens was used as the templates for reverse-transcription polymerase chain reaction. Relative quantitative analysis of TYMP gene mRNA expression was implemented using the LightCycler ${ }^{\circledR} 480$ (Roche, Shanghai, China) with SYBR Premix EX Taq system. The forward primer of TYMP was 5'-ATGGCAGCCTTGATGACC-3', the reverse primer was 5'- TTATTGCTGCGGCGGCAG-3'. Amplification system was comprised of $10 \mu$ l SYBR Premix EX Taq, $0.2 \mu$ l of each primer $(20 \mu \mathrm{M}), 7.6 \mu \mathrm{l}$ double distilled water (ddH2O) and $2 \mu \mathrm{l}$ cDNA. TYMP gene mRNA expression was determined by the method of comparative $\mathrm{Ct}\left(2^{-} \Delta \mathrm{Ct}\right)$ with GAPDH mRNA expression being used as an endogenous control according to the previous research[18].

\section{Statistical analysis}

Correlation analysis were performed using SPSS version 25.0 (IBM, USA). Hardy-Weinberg equilibrium test was performed for the rs 11479 genotypes status using $\chi^{2}$ test method. The significance of proportion variables and continuous variables according to rs11479 genotype status was identified using the $\chi^{2}$ test and the Mann-Whitney U nonparametric test (between the two groups), respectively. The primary analysis of present study was the association between DFS or OS and rs 11479 genotype status. Survival curves were generated using Stata 14.0 to present DFS and OS data according to rs 11479 genotype status with log-rank test to estimate the significant difference. DFS was defined as the time from the onset of surgical resection to the patients' recurrence of disease or death from any cause. Overall survival (OS) was defined as the time from the onset of surgical resection to the date of patients' death from any cause. Those without death event by the end of the study follow-up, survival end points were censored at the date of last follow-up. Additionally, Cox multivariable analysis was introduced for DFS. $P<0.05$ was accepted as statistical significance.

\section{Results}

\section{Baseline characteristics of the 218 patients with CRC according to TYMP rs11479 genotype status}


Baseline characteristics of the 218 patients with CRC included in present study were exhibited in Table 1. The median age was 59 years (range: 28-83 years). Male and female was observed in 127 and 91 patients, respectively. ECOG performance status score of 0 and 1-2 score was noted in 148 and 70 patients, respectively. Besides, color cancer and rector cancer were seen in 153 and 65 patients. Furthermore, patients with pathological staging of $\otimes$ and $\otimes$ was observed in 170 and 48 patients, respectively. And majority histological type was adenocarcinoma with 196 patients. Regarding the mismatch repair gene (MMR) status, there were 15 patients $(11.0 \%)$ with deficiency of mismatch repair (dMMR) and 109 patients with proficiency of mismatch repair (pMMR). However, a total of 94 patients were not available for MMR status. Additionally, a total of 47 patients $(21.6 \%)$ received adjuvant radiotherapy along with adjuvant chemotherapy. Furthermore, capecitabine monotherapy was administered in 53 patients and CAPEOX (capecitabine combined with oxaliplatin) regimen was implemented in 165 patients. Interestingly, it should be noted that he actual median completion cycle of capecitabine monotherapy regimen was 5 cycles (range: 3-8 cycles). And the actual median completion cycle of CAPEOX regimen was 4 cycles (range: 2-8 cycles). 
Table 1

Baseline characteristics of the 218 patients with CRC according to TYMP rs 11479 genotype status

\begin{tabular}{|c|c|c|c|c|c|}
\hline \multirow[t]{2}{*}{ Baseline characteristics } & \multirow[t]{2}{*}{$\begin{array}{l}\text { Total }(\mathrm{N}=218 \text {, } \\
\%)\end{array}$} & \multicolumn{2}{|c|}{$\begin{array}{l}\text { TYMP rs } 11479 \text { genotype } \\
\text { status }\end{array}$} & \multirow[t]{2}{*}{$\chi^{2}$} & \multirow[t]{2}{*}{$P$} \\
\hline & & $\begin{array}{l}\mathrm{GG} \\
(\mathrm{N}=141)\end{array}$ & $\begin{array}{l}\text { GAVAA } \\
(\mathrm{N}=77)\end{array}$ & & \\
\hline Age & $59(28-83)$ & $59(31-83)$ & $59(28-81)$ & NA & 0.519 \\
\hline \multicolumn{6}{|l|}{ Median (range) } \\
\hline Gender & $127(58.3)$ & $84(59.6)$ & $43(55.8)$ & 0.285 & 0.593 \\
\hline Male & $91(41.7)$ & $57(40.4)$ & $34(44.2)$ & & \\
\hline \multicolumn{6}{|l|}{ Female } \\
\hline ECOG PS score & 148 (67.9) & $92(65.2)$ & $56(72.7)$ & 1.278 & 0.258 \\
\hline 0 & $70(32.1)$ & $49(34.8)$ & $21(27.3)$ & & \\
\hline \multicolumn{6}{|l|}{$1-2$} \\
\hline Tumor location & $153(70.2)$ & $99(70.2)$ & $54(70.1)$ & 0.000 & 0.990 \\
\hline Color cancer & $65(29.8)$ & $42(29.8)$ & $23(29.9)$ & & \\
\hline \multicolumn{6}{|l|}{ Rector cancer } \\
\hline Pathological staging & $48(22.0)$ & $28(19.8)$ & $20(26.0)$ & 1.085 & 0.298 \\
\hline ૧ & $170(78.0)$ & $113(80.2)$ & $57(74.0)$ & & \\
\hline \multicolumn{6}{|l|}{ प } \\
\hline Histological type & 196 (89.9) & 125 (88.7) & $71(92.2)$ & 0.694 & 0.405 \\
\hline Adenocarcinoma & $22(10.1)$ & $16(11.3)$ & $6(7.8)$ & & \\
\hline \multicolumn{6}{|l|}{ Other type } \\
\hline MMR status & $15(6.9)$ & $9(6.4)$ & $6(7.8)$ & 0.156 & 0.925 \\
\hline dMMR & $109(50.0)$ & $71(50.4)$ & $38(49.4)$ & & \\
\hline pMMR & $94(43.1)$ & $61(43.3)$ & $33(42.8)$ & & \\
\hline NA & & & & & \\
\hline
\end{tabular}

Abbreviation: CRC: colorectal cancer; TYMP: thymidine phosphorylase; ECOG: Eastern Cooperative Oncology Group; PS: performance status; MMR: mismatch repair; dMMR: deficiency of mismatch repair; pMMR: proficiency of mismatch repair; CAPEOX: capecitabine plus oxaliplatin; NA: not available. 


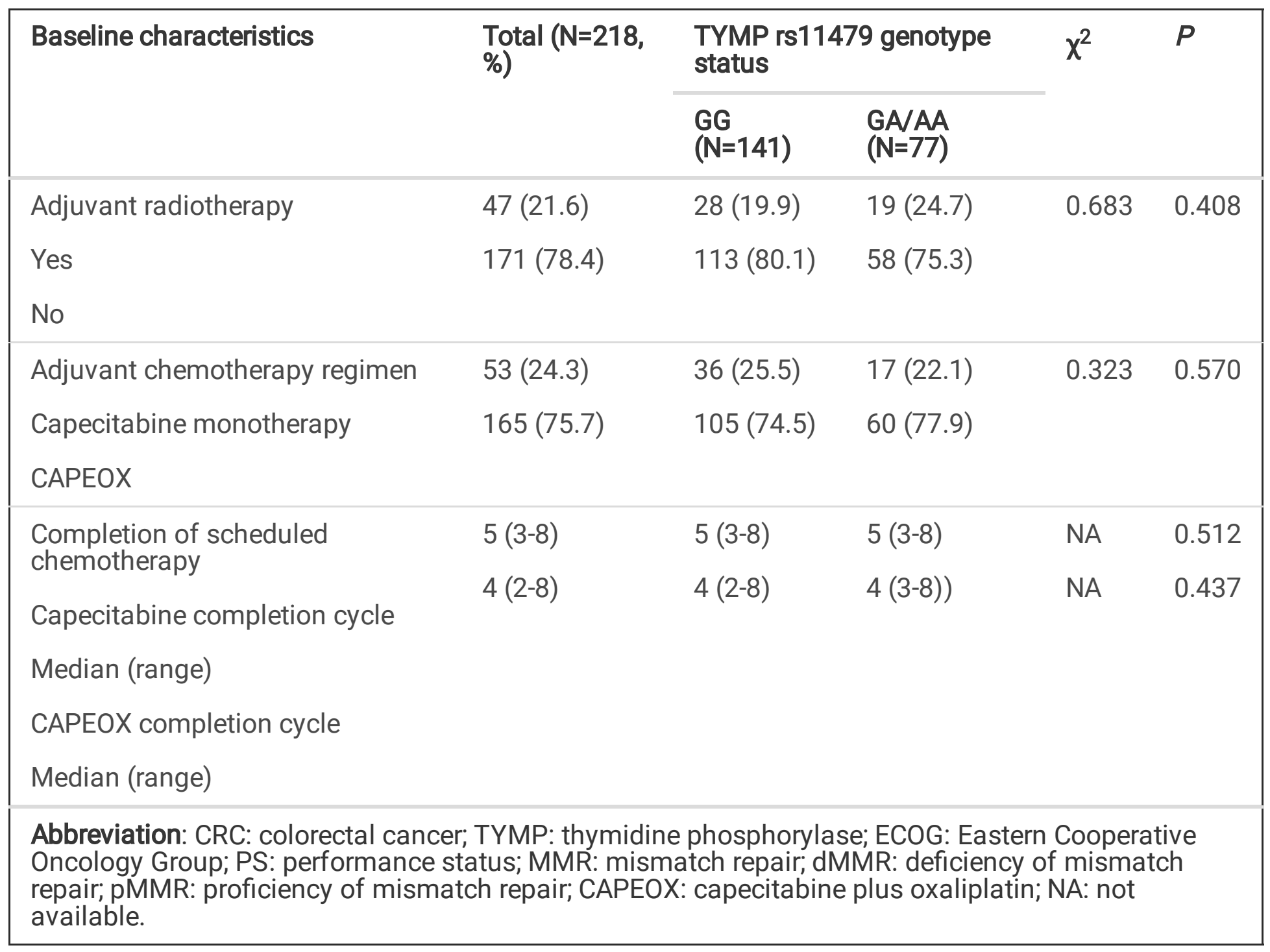

As described in the method part, only TYMP rs11479 was of clinical significance preliminarily. The prevalence of rs 11479 polymorphism among the 218 patients with CRC manifested as: GG genotype 141 cases (64.7\%), GA genotype 68 cases (31.2\%), AA genotype 9 cases (4.1\%), minor allele frequency of rs 11479 was 0.20 . And the distribution of the three genotypes was in accordance with Hardy-Weinberg Equilibrium ( $P=0.825)$. Given that the frequency of AA genotype was relatively rare, patients with $A A$ and GA genotypes were merged in the subsequent analysis. As presented in Table 2, patients with GG and GA/AA genotype were well balanced with similar baseline characteristics and no statistically significant difference was observed $(P>0.05)$. 
Table 2

Univariate and multivariate analyses of DFS among the 218 patients with colorectal cancer

\begin{tabular}{|c|c|c|c|c|}
\hline \multirow[t]{2}{*}{ Baseline characteristics } & \multirow{2}{*}{$\begin{array}{l}\text { Median DFS } \\
(95 \% \mathrm{Cl})\end{array}$} & \multirow{2}{*}{$\begin{array}{l}P \text { (univariate } \\
\text { analysis) }\end{array}$} & \multicolumn{2}{|c|}{ Multivariate analysis } \\
\hline & & & $\mathrm{HR}(95 \% \mathrm{Cl})$ & $\mathbf{P}$ \\
\hline Age & $5.30(4.50-6.10)$ & 0.313 & & \\
\hline$<59$ & $4.50(3.65-5.35)$ & & & \\
\hline \multicolumn{5}{|l|}{$\geq 59$} \\
\hline Gender & $5.50(4.59-6.41)$ & 0.435 & & \\
\hline Female & $4.55(3.72-5.38)$ & & & \\
\hline \multicolumn{5}{|l|}{ Male } \\
\hline ECOG PS score & $5.80(4.93-6.67)$ & 0.009 & $0.65(0.46-$ & 0.015 \\
\hline 0 & $3.89(3.11-4.67)$ & & & \\
\hline \multicolumn{5}{|l|}{$1-2$} \\
\hline Tumor location & $5.10(4.28-5.92)$ & 0.531 & & \\
\hline Color cancer & $4.10(5.25-4.95)$ & & & \\
\hline \multicolumn{5}{|l|}{ Rector cancer } \\
\hline Pathological staging & $6.15(5.09-7.21)$ & 0.002 & $0.57(0.33-$ & 0.007 \\
\hline ; & $3.89(3.02-4.76)$ & & & \\
\hline \multicolumn{5}{|l|}{ ] } \\
\hline Histological type & $5.10(4.08-6.12)$ & 0.461 & & \\
\hline Adenocarcinoma & $4.30(3.55-5.05)$ & & & \\
\hline \multicolumn{5}{|l|}{ Other type } \\
\hline MMR status & $4.10(3.24-4.96)$ & 0.614 & & \\
\hline dMMR & $4.89(4.02-5.76)$ & & & \\
\hline \multicolumn{5}{|l|}{ Other status } \\
\hline Adjuvant radiotherapy & $5.00(4.09-5.91)$ & 0.619 & & \\
\hline Yes & $4.30(3.51-5.09)$ & & & \\
\hline \multicolumn{5}{|l|}{ No } \\
\hline $\begin{array}{l}\text { Abbreviation: CRC: colore } \\
\text { ECOG: Eastern Cooperati } \\
\text { deficiency of mismatch } r \\
\text { hazard ratio. }\end{array}$ & $\begin{array}{l}\text { Incer; DFS: diseas } \\
\text { ology Group; PS: } \\
\text { AAPEOX: capecitak }\end{array}$ & $\begin{array}{l}\text { e survival; TYN } \\
\text { rmance status } \\
\text { plus oxaliplatir }\end{array}$ & $\begin{array}{l}\text { dine phospho } \\
\text { ismatch repa } \\
\text { fidence interv }\end{array}$ & $\begin{array}{l}\text { Re; } \\
\text { Mír: }\end{array}$ \\
\hline
\end{tabular}




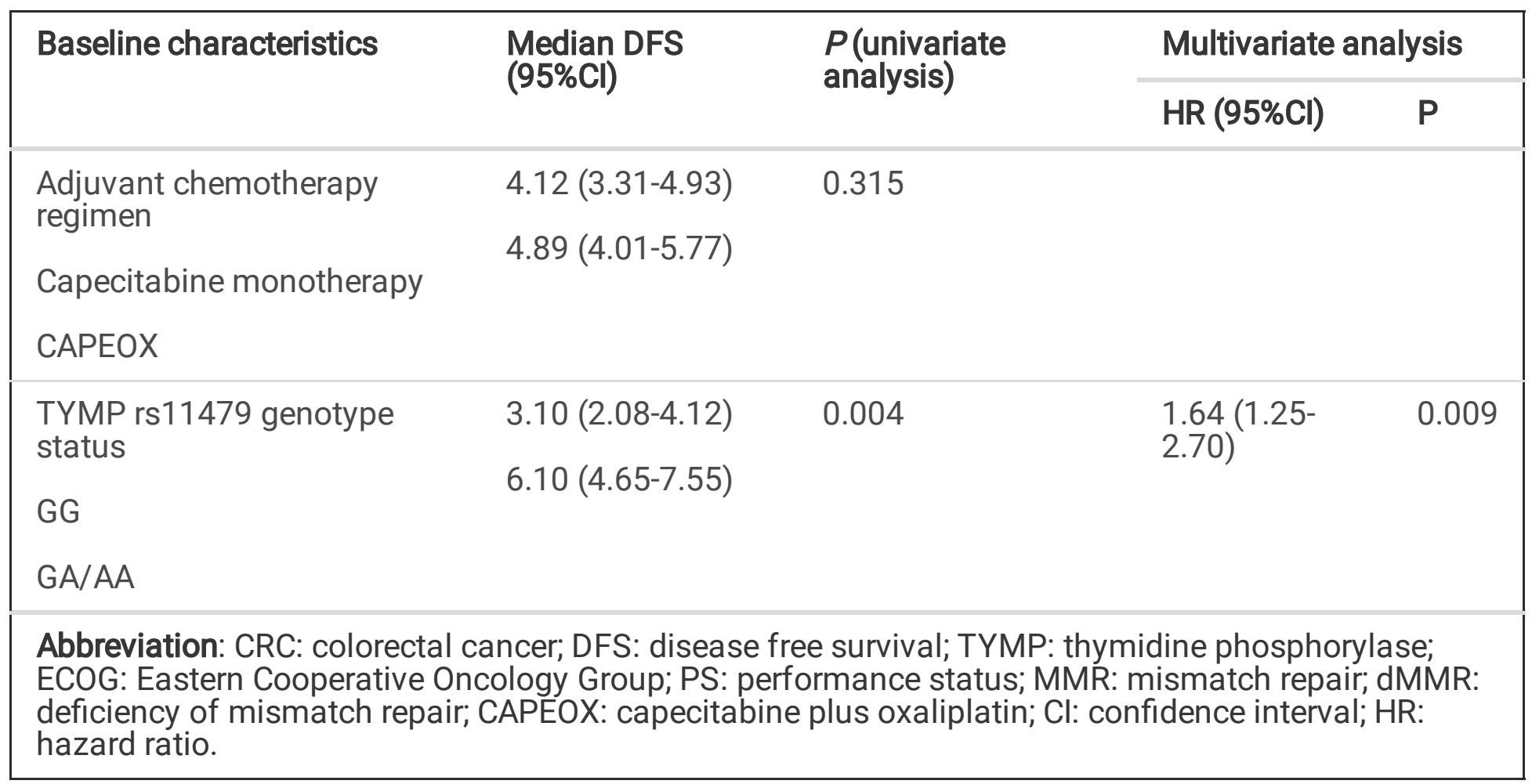

\section{Implications of TYMP rs11479 polymorphism on the prognosis of the 218 patients with CRC}

All the 218 patients with CRC included in this study were available for prognostic assessment. And the last follow-up date of present study was September 2021. The median follow-up duration from the patients included in this study to last follow-up date was 5.5 years (follow-up range: 0.25-10 years). Collectively, the prognostic data of the 218 patients with CRC was presented in Figure 3 and Figure 4 . As shown in Figure 3, The median DFS of the 218 patients was 4.6 years [95\% confidence interval $(\mathrm{Cl})$ : 3.80 5.40], 3-year DFS rate was 59.04\% (95\%Cl: $52.13 \%-65.29 \%)$ and 5-year DFS rate was $45.95 \%(95 \% \mathrm{Cl}$ : $38.89 \%-52.71 \%)$, respectively. Furthermore, as illustrated in Figure 4, the median OS of the 218-patient cohort was 5.8 years (95\% Cl: 5.12-6.48), the 3-year OS rate was $78.76 \%(95 \% \mathrm{Cl}$ : $72.60 \%-83.70 \%)$ and 5year OS rate was $54.60 \%$ (95\%Cl: $47.29 \%-61.32 \%)$.

In order to identify the clinical significance of TYMP rs11479 polymorphism, association analysis between genotype status of rs11479 and DFS was performed firstly. As exhibited in Figure 4, the median DFS of the patients with $\mathrm{GG}$ and GA/AA genotype of rs 11479 polymorphism was 3.1 months $(95 \% \mathrm{Cl}$ : 2.08-4.12) and 6.1 (95\% Cl: 4.65-7.55) months, respectively. And the 3-year DFS rate was $50.38 \%(95 \% \mathrm{Cl}$ : $41.78 \%-58.36 \%)$ and $74.97 \%(95 \% \mathrm{Cl}: 63.58 \%-83.25 \%)$, which was statistically significant difference $\left(\chi^{2}=\right.$ $8.164, P=0.004)$. Furthermore, regarding the association between rs11479 genotype status and OS, as illustrated in Figure 5, the median OS of the patients with GG and GA/AA genotype of rs 11479 polymorphism was 5.0 (95\% Cl: 4.05-5.95) months and 7.0 (95\%Cl: NA-NA) months, respectively. The 5year OS rate of patients with GG and GA/AA genotype of rs 11479 polymorphism was $49.65 \%$ ( $95 \% \mathrm{Cl}$ : 40.52\%-58.11\%) and $63.31 \%$ (95\%Cl: 50.83\%-73.44\%). And the difference was statistically significant $\left(\chi^{2}\right.$ $=4.562, P=0.033)$. 
Furthermore, the median DFS according to different baseline characteristic subgroups in univariate analysis were performed simultaneously. As exhibited in Table 2, only ECOG PS score and pathological staging was significantly associated with DFS in univariate analysis according to baseline characteristics, which suggested that the median DFS of patients with ECOG 0 score was dramatically longer than that of patients with the $1-2$ score (median DFS: 5.80 vs 3.89 months, $P=0.009$ ), the median

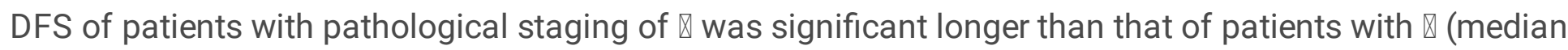
DFS: 6.15 vs 3.89 months, $P=0.002$ ). Furthermore, a multivariate Cox regression model was introduced for DFS adjustment including the baseline characteristics which was significant in the univariate analysis as illustrated in Table 2. The multivariate analysis results were illustrated in Table 2, after the multivariate adjustment, the significant statistical difference was still observed regarding TYMP rs11479 polymorphism for DFS, which suggested that rs 11479 polymorphism was an independent factor for DFS [hazard ratio $(H R)=1.64, P=0.009$ ). Besides, as presented in Table 2 , after adjusted in the Cox regression analysis, both $E C O G$ score $(H R=0.65, P=0.015)$ and pathological staging $(H R=0.57, P=0.007)$ were independent factors for DFS.

\section{Relevance Of Rs11479 Polymorphism To Tymp Gene Mrna Expression}

As mentioned in the methods part, a total of 65 PBMC specimens were available for TYMP gene mRNA expression analysis finally. The expression of TYMP gene mRNA was detected by extracting RNA from the 65 PBMC specimens and the relevance analysis between the genotype status of rs11479 polymorphism and TYMP mRNA expression was analyzed accordingly. Firstly, the genotype status of rs 11479 polymorphism in the 65 PBMC specimens manifested as: GG genotype 42 cases (64.6\%), GA genotype 20 cases (30.8\%) and AA genotype 3 cases (4.6\%). The MAF was 0.20 and distribution frequency of the three genotypes was in accordance with the Hardy-Weinberg equilibrium $(P=0.756)$ as well, which were comparable to the genotype distribution among the 218 patients with CRC. Similarly, GA and AA genotypes were merged in the subsequent analysis. As illustrated in Figure 6 , the relative expression of TYMP mRNA in PBMC of patients with GA/AA genotype was significantly higher than that of patients with GG genotype of rs 11479 polymorphism (3.945 \pm 0.575 vs $3.191 \pm 0.726)$, which was statistically significant $(\mathrm{t}=4.294, P<0.001)$.

\section{Discussion}

Firstly, present study provided and highlighted the real-world evidence regarding the prognostic data of patients with CRC who were treated with surgical resection and capecitabine-based adjuvant chemotherapy in real-world. Simultaneously, the prognostic association analysis suggested that TYMP gene polymorphism rs11479 might involve in the prognosis of postoperative patients with CRC who received capecitabine based adjuvant chemotherapy through the mediation TYMP mRNA expression. 
To the best of our knowledge, CRC was established to be a highly heterogeneous digestive system malignancy[19]. Comparatively limited research progresses that could strikingly improve the prognosis of the patients with CRC were observed in recent years[20]. At present, PD-1/PD-L1 blockades were reported to be effective for considerable patients with advanced stage of cancer which provided significant survival benefit to some extent[21]. Nevertheless, the availability of PD-1/PD-L1 blockades in advanced CRC was scanty, which indicated that only patients with dMMR might benefit from PD-1 blockade according to Keynote 177 clinical trial[22]. As a result, traditional chemotherapy still played an important role in the treatment for CRC. However, it should be noticed that ORR of chemotherapy was relatively limited, which exhibited the urgent exploration of potential biomarkers that could predict the efficacy of traditional chemotherapy was an important research direction[23]. At present, biomarkers that might predict the effectiveness to traditional chemotherapy were predominately focused on genetic polymorphisms, ctDNA and somatic gene mutations[24]. Specifically, a recently reported polymorphism study initiated by JS Su and colleagues indicated that PD-L1 gene polymorphism 901T >C could be used as a potential biomarker to involve in the prognosis of patients with $C R C$ receiving capecitabine-based adjuvant chemotherapy through mediation of the mRNA expression of PD-L1[25]. Furthermore, another study initiated by JT and colleagues investigated the clinical significance of ctDNA for guiding the prognosis of patients with high-risk stage $\otimes$ colon cancer, which indicated that the monitoring of ctDNA might be useful to predict the prognosis of patients with high-risk stage $₫$ colon cancer[26]. Additionally, AJ Li and colleagues performed a study to explore the predictive association between PIK3CA and TP53 somatic mutations status and OS for patients with stage $\otimes$ and $\otimes C R C[27]$. And the results suggested that patients with PIK3CA and TP53 double mutations were correlated with worse OS. Collectively, all the above findings demonstrated that genome DNA status of colon cancer or rectal cancer might predict the prognosis of patients with CRC who were treated with conventional chemotherapy to some extent[28].

Although present study was designed as a retrospective analysis, we still carried out the prognostic analysis of the 218 patients with CRC who received capecitabine-based adjuvant chemotherapy. And the results exhibited that the median DFS of the 218 patients was 4.6 years $(95 \% \mathrm{Cl}$ : $3.80-5.40)$ and the median OS of the 218-patient cohort was 5.8 years (95\%Cl: $5.12-6.48)$. The DFS and OS data in our study seemed to be lower than that in N016968 clinical trial which was implemented to identify the feasibility of oxaliplatin combined with capecitabine as adjuvant therapy for stage $\nabla$ CRC[29]. The discrepancy between the two study could be attributed to the following explanation: our study included more patients with an ECOG performance status of 2 score than that in the N016968 study and the results of the Cox analysis in our study indicated that patients with ECOG of 2 score conferred a worse prognosis, which was consistent with the previous study[30]. Besides, it should be noted that this study was designed as a retrospective analysis. Management of the patients in retrospective study was not sufficient and normative compared with well-designed phase $\otimes$ clinical trial, which was also reflected in the fact that the actual completion of capecitabine monotherapy regimen in this study was only 5 cycles, and the CAPEOX regimen was only 4 cycles. The insufficient adjuvant chemotherapy might stand a good chance to compromise the survival of the patients[31]. 
Considerable previous studies indicated that genetic variation of drug metabolism gene might contribute to the effectiveness of a variety of drugs[32]. To our knowledge, our study might be the first exploration disclosed that the carriers of the T allele at rs11479 of TYMP gene could be sensitive to capecitabine administration and benefit from capecitabine adjuvant chemotherapy in Chinese patients with CRC who received capecitabine based adjuvant chemotherapy by influencing the mRNA expression of TYMP. Interestingly, a previous study initiated by YB Du and colleagues was reported[15]. A total of 235 patients with CRC underwent surgical treatment were included in their study retrospectively and they investigated the influence of TYMP genetic variation on clinical outcomes of patients with CRC. The conclusion in their study suggested that 5633C>T of TYMP might impact the prognosis of the patients, which was consistent with the design and results of our study. However, the heterogeneous adjuvant chemotherapy regimens might compromise the clinical significance of 5633C>T of TYMP in their study. Another recent study initiated by WC Chen and colleagues performed the implication of TYMP genetic variation on the clinical outcome of gastric cancer patients received capecitabine based adjuvant chemotherapy[17]. A total of 198 patients with gastric cancer were participated in the study and clinical significance of TYMP genetic variation was implemented. Furthermore, the conclusion exhibited that rs 11479 of TYMP had favorable influence on the clinical outcomes of gastric cancer patients received capecitabine based adjuvant chemotherapy, which was in concert with our study even the tumors in the two studies were different. On other hand, another study initiated by $\mathrm{H}$ Liu and colleagues investigated the relevance of TYMP genetic variation to the survival of gastrointestinal cancer patients treating by fluoropyrimidines[16]. A total of 141 metastatic GIC patients received 5-FU based first-line chemotherapy were included and the results suggested that $T$ allele gene carriers were associated with higher TYMP gene expression, which was consistent with the mRNA expression results in our study. However, they failed to identify the positive association of the polymorphism with OS mainly own to the relatively limited sample size. Furthermore, a previous study initiated by BA Jennings and colleagues found that $T$ allele gene carriers of rs 11479 were correlated with higher incidence of adverse reaction among patients with $\mathrm{CRC}$ receiving capecitabine therapy, which was partly in line with the results of our study, highlighting the possibility that $T$ allele carriers of rs11479 might be more sensitive to capecitabine administration[33].

Interestingly, the relationship between the expression level of TYMP gene and the prognosis of CRC remains controversial in recent study [34]. Our study preliminarily sugested that patients with higher mRNA expression of TYMP might be likely to benefit from capecitabine administration, thus conferring a superior prognosis, which was consistent with the results of the previous study initiated by M Lu and colleagues[35]. A total of 57 patients with advanced gastric cancer who received capecitabine-based regimen were included and identified that the MRNA expression of TYMP was positively associated with overall response and OS. Nevertheless, on the other hand, considerable studies had found that TYMP gene was an important factor promoting tumor angiogenesis, which demonstrated that higher expression of TYMP gene was usually accompanied by a higher tumor burden, which generated the tendency that the tumor was easy to recurrence and metastasis, thus contributing to the worse prognosis [36]. Collectively, TYMP gene played a dual role in vivo. On the one hand, it stimulated angiogenesis of tumor 
cells to promote tumor growth. On the other hand, TYMP was an important metabolic gene of capecitabine and a key gene for 5-FU chemotherapy drugs to play a cytotoxic role and kill tumor cells[37]. In a word, the conclusion in our study was needed to be confirmed in large-scale clinical trials subsequently.

The limitations of this study were as follows: firstly, the sample size of the study was relatively small, and the clinical significance of TYMP polymorphism in patient with CRC was still needed to be evaluated in a in a larger population. Secondly, present study was designed as a retrospective analysis and some bias could not be avoided. However, the clinical significance of TYMP rs11479 was fully evaluated, we thought our study was of clinical significance for the prognostic evaluation of patients with CRC who received surgical resection and capecitabine-based adjuvant chemotherapy.

\section{Conclusions}

Collectively, present study provided real-world evidence regarding the prognostic data of patients with CRC who were treated with surgical resection and capecitabine-based adjuvant chemotherapy in realworld. Simultaneously, the prognostic association analysis suggested that TYMP gene polymorphism rs11479 might involve in the prognosis of patients with CRC who received capecitabine based adjuvant chemotherapy through mediation of the mRNA expression of TYMP.

\section{Abbreviations}

CRC

Colorectal cancer

TYMP

Thymidine phosphorylase

PBMC

Peripheral blood mononuclear cell

SNP

Single-nucleotide polymorphisms

\section{Declarations}

\section{Acknowledgements}

The authors would like to express sincere gratitude to the patients and their families for participating in this study. We would thank all the staff who took part in this study.

\section{Authors' contributions}

Xinyu Peng and Tao Zhang designed the study and prepared this manuscript. Junjie Sun, Hengxue Lin and Tianliang Bai collected the data and conducted the statistics. Yating Qiao, Yaxin Li, Gang Li and 
Guicun Li participated in the experiments and follow-up. Xiongjie Jia and Aimin Zhang supervised the study and edited the manuscript. All authors participated in the reviewed of the manuscript and approved the final manuscript.

\section{Funding}

The authors received no specific funding for this work.

\section{Availability of data and materials}

The analyzed datasets generated during the study are possibly available from the corresponding author on reasonable request.

\section{Ethics approval and consent to participate}

The study protocol was approved by the ethics committee of the Affiliated Hospital of Hebei University.

\section{Consent for publication}

Not applicable.

\section{Competing interests}

The authors declare that they have no competing interests.

\section{References}

1. Wong MCS, Huang J, Lok V, et al. Differences in Incidence and Mortality Trends of Colorectal Cancer Worldwide Based on Sex, Age, and Anatomic Location. Clin Gastroenterol Hepatol. 2021;19:95566.e961.

2. Koo CY, Tai BC, Chan DKH, et al. Chemotherapy and adverse cardiovascular events in colorectal cancer patients undergoing surgical resection. World J Surg Oncol. 2021;19:21.

3. Koulis C, Yap R, Engel R, et al. Personalized Medicine-Current and Emerging Predictive and Prognostic Biomarkers in Colorectal Cancer. Cancers (Basel). 2020; 12.

4. Biller LH, Schrag D. Diagnosis and Treatment of Metastatic Colorectal Cancer: A Review. Jama. 2021;325:669-85.

5. Grothey A, Sobrero AF, Shields AF, et al. Duration of Adjuvant Chemotherapy for Stage III Colon Cancer. N Engl J Med. 2018;378:1177-88.

6. Yamazaki K, Yamanaka T, Shiozawa M, et al. Oxaliplatin-based adjuvant chemotherapy duration (3 versus 6 months) for high-risk stage II colon cancer: the randomized phase III ACHIEVE-2 trial. Ann Oncol. 2021;32:77-84. 
7. Dasari A, Morris VK, Allegra CJ, et al. ctDNA applications and integration in colorectal cancer: an $\mathrm{NCl}$ Colon and Rectal-Anal Task Forces whitepaper. Nat Rev Clin Oncol. 2020;17:757-70.

8. Osumi H, Shinozaki E, Yamaguchi K, et al. Clinical utility of circulating tumor DNA for colorectal cancer. Cancer Sci. 2019;110:1148-55.

9. Hamaguchi R, Tsuchiya T, Miyata G, et al. Efficacy of oral administration of cystine and theanine in colorectal cancer patients undergoing capecitabine-based adjuvant chemotherapy after surgery: a multi-institutional, randomized, double-blinded, placebo-controlled, phase II trial (JORTC-CAM03). Support Care Cancer. 2020;28:3649-57.

10. Yang $L$, Sun $Y$, Huang $X E$, et al. Carcinoma microsatellite instability status as a predictor of benefit from fluorouracil-based adjuvant chemotherapy for stage II rectal cancer. Asian Pac J Cancer Prev. 2015;16:1545-51.

11. Lam SW, Guchelaar HJ, Boven E. The role of pharmacogenetics in capecitabine efficacy and toxicity. Cancer Treat Rev. 2016;50:9-22.

12. Mitselou A, loachim E, Skoufi U, et al. Predictive role of thymidine phosphorylase expression in patients with colorectal cancer and its association with angiogenesis-related proteins and extracellular matrix components. In Vivo. 2012;26:1057-67.

13. Won HS, Lee MA, Chung ES, et al. Comparison of thymidine phosphorylase expression and prognostic factors in gallbladder and bile duct cancer. BMC Cancer. 2010;10:564.

14. Amatori F, Di Paolo A, Del Tacca M, et al. Thymidylate synthase, dihydropyrimidine dehydrogenase and thymidine phosphorylase expression in colorectal cancer and normal mucosa in patients. Pharmacogenet Genomics. 2006;16:809-16.

15. Du YB, Zhang TF, Cui K, et al. [The influence of Thymidine Phosphorylase genetic variation on clinical outcomes and safety of colorectal cancer patients received adjuvant chemotherapy after R0 resection]. Zhonghua Yi Xue Za Zhi. 2018;98:2569-73.

16. Huang $L$, Chen $F$, Chen $Y$, et al. Thymidine phosphorylase gene variant, platelet counts and survival in gastrointestinal cancer patients treated by fluoropyrimidines. Sci Rep. 2014;4:5697.

17. Chen WC, Wu G, Zhang W, et al. [Clinical outcomes of gastric cancer patients received capecitabine based adjuvant chemotherapy and the corresponding pharmacogenomics analysis]. Zhonghua Yi Xue Za Zhi. 2018;98:3420-5.

18. Song ZZ, Zhao LF, Zuo J, et al. Clinical Outcomes and Safety of Apatinib Mesylate in the Treatment of Advanced Non-Squamous Non-Small Cell Lung Cancer in Patients Who Progressed After Standard Therapy and Analysis of the KDR Gene Polymorphism. Onco Targets Ther. 2020;13:603-13.

19. Zhang Y, Song J, Zhao Z, et al. Single-cell transcriptome analysis reveals tumor immune microenvironment heterogenicity and granulocytes enrichment in colorectal cancer liver metastases. Cancer Lett. 2020;470:84-94.

20. Hermann, Brenner. Chen. The colorectal cancer epidemic: challenges and opportunities for primary, secondary and tertiary prevention. British Journal of Cancer. 2018. 
21. Ai L, Chen J, Yan H, et al. Research Status and Outlook of PD-1/PD-L1 Inhibitors for Cancer Therapy. Drug Des Devel Ther. 2020;14:3625-49.

22. André T, Shiu KK, Kim TW, et al. Pembrolizumab in Microsatellite-Instability-High Advanced Colorectal Cancer. N Engl J Med. 2020;383:2207-18.

23. Malla SB, Fisher DJ, Domingo E, et al. In-depth Clinical and Biological Exploration of DNA Damage Immune Response as a Biomarker for Oxaliplatin Use in Colorectal Cancer. Clin Cancer Res. 2021;27:288-300.

24. Reece $\mathrm{M}$, Saluja $\mathrm{H}$, Hollington $\mathrm{P}$, et al. The Use of Circulating Tumor DNA to Monitor and Predict Response to Treatment in Colorectal Cancer. Front Genet. 2019;10:1118.

25. Su J, Dai B, Yuan W, et al. The influence of PD-L1 genetic variation on the prognosis of R0 resection colorectal cancer patients received capecitabine-based adjuvant chemotherapy: a long-term followup, real-world retrospective study. Cancer Chemother Pharmacol. 2020;85:969-78.

26. Tie J, Cohen JD, Wang Y, et al. Circulating Tumor DNA Analyses as Markers of Recurrence Risk and Benefit of Adjuvant Therapy for Stage III Colon Cancer. JAMA Oncol. 2019;5:1710-7.

27. Li AJ, Li HG, Tang EJ, et al. PIK3CA and TP53 mutations predict overall survival of stage II/III colorectal cancer patients. World J Gastroenterol. 2018;24:631-40.

28. Ogunwobi OO, Mahmood F, Akingboye A. Biomarkers in Colorectal Cancer: Current Research and Future Prospects. Int J Mol Sci. 2020; 21.

29. Schmoll HJ, Tabernero J, Maroun J, et al. Capecitabine Plus Oxaliplatin Compared With Fluorouracil/Folinic Acid As Adjuvant Therapy for Stage III Colon Cancer: Final Results of the N016968 Randomized Controlled Phase III Trial. J Clin Oncol. 2015;33:3733-40.

30. Yang J, Xu H, Guo X, et al. Pretreatment Inflammatory Indexes as Prognostic Predictors for Survival in Colorectal Cancer Patients Receiving Neoadjuvant Chemoradiotherapy. Sci Rep. 2018;8:3044.

31. Iveson TJ, Sobrero AF, Yoshino T, et al. Duration of Adjuvant Doublet Chemotherapy (3 or 6 months) in Patients With High-Risk Stage II Colorectal Cancer. J Clin Oncol. 2021;39:631-41.

32. de Man FM, Goey AKL, van Schaik RHN, et al. Individualization of Irinotecan Treatment: A Review of Pharmacokinetics, Pharmacodynamics, and Pharmacogenetics. Clin Pharmacokinet. 2018;57:122954.

33. Jennings BA, Loke YK, Skinner J, et al. Evaluating predictive pharmacogenetic signatures of adverse events in colorectal cancer patients treated with fluoropyrimidines. PLoS One. 2013;8:e78053.

34. Marangoni E, Laurent C, Coussy F, et al. Capecitabine Efficacy Is Correlated with TYMP and RB1 Expression in PDX Established from Triple-Negative Breast Cancers. Clin Cancer Res. 2018;24:260515.

35. Lu M, Gao J, Wang XC, et al. Expressions of Thymidylate Synthase, Thymidine Phosphorylase, Class III beta-tubulin, and Excision Repair Cross-complementing Group 1 predict Response in Advanced Gastric Cancer Patients Receiving Capecitabine Plus Paclitaxel or Cisplatin. Chin J Cancer Res. 2011;23:288-94. 
36. Huang X, Wang L, Chen Y, et al. Poor Prognosis Associated with High Levels of Thymidine Phosphorylase and Thrombocytosis in Patients with Renal Cell Carcinoma. Urol Int. 2017;98:162-8.

37. Bronckaers A, Gago F, Balzarini J, et al. The dual role of thymidine phosphorylase in cancer development and chemotherapy. Med Res Rev. 2009;29:903-53.

\section{Figures}

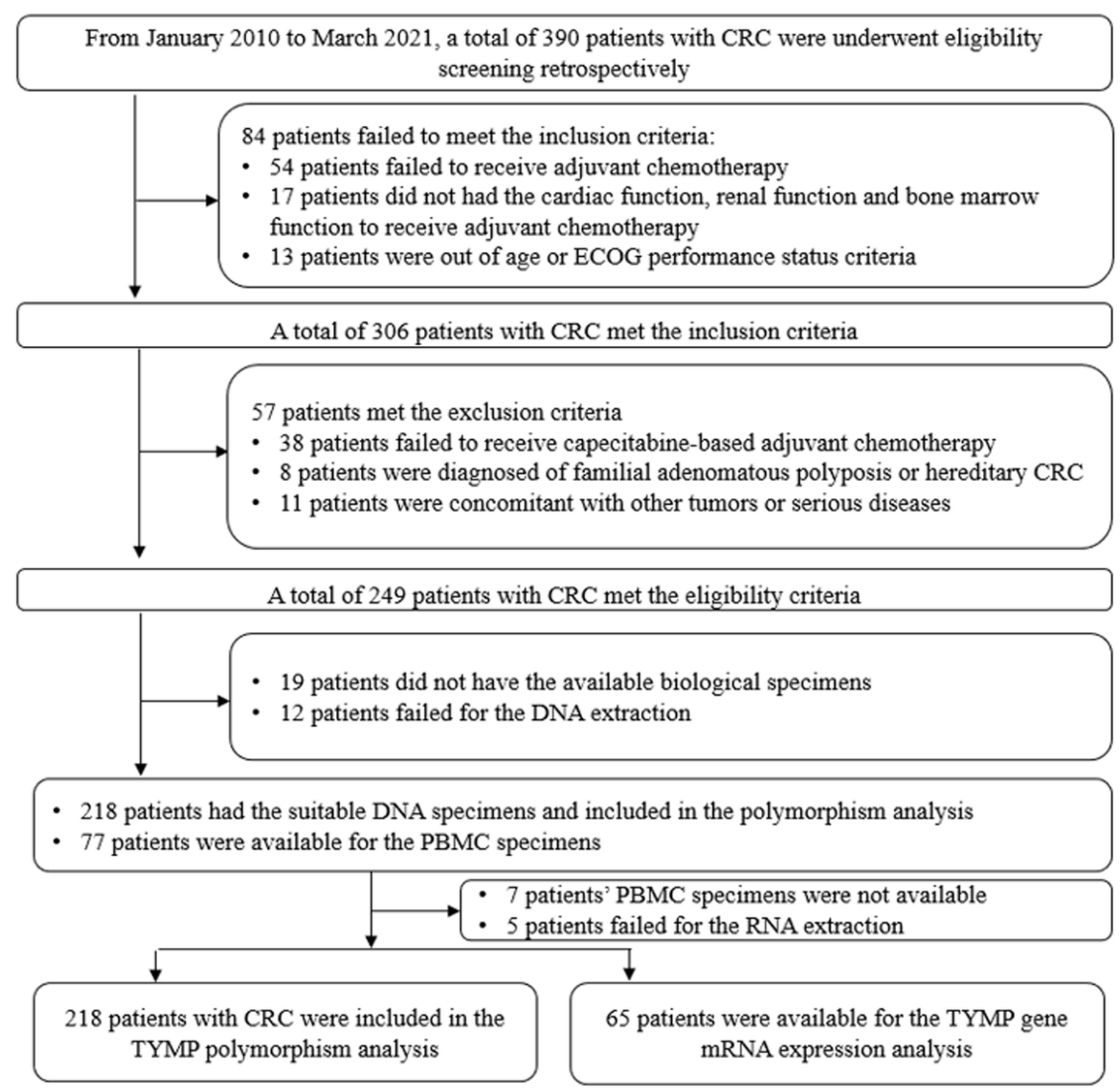

Figure 1 
Flow chart of this retrospective study of influence of TYMP polymorphism on the prognosis of patients with colorectal cancer receiving capecitabine-based adjuvant chemotherapy

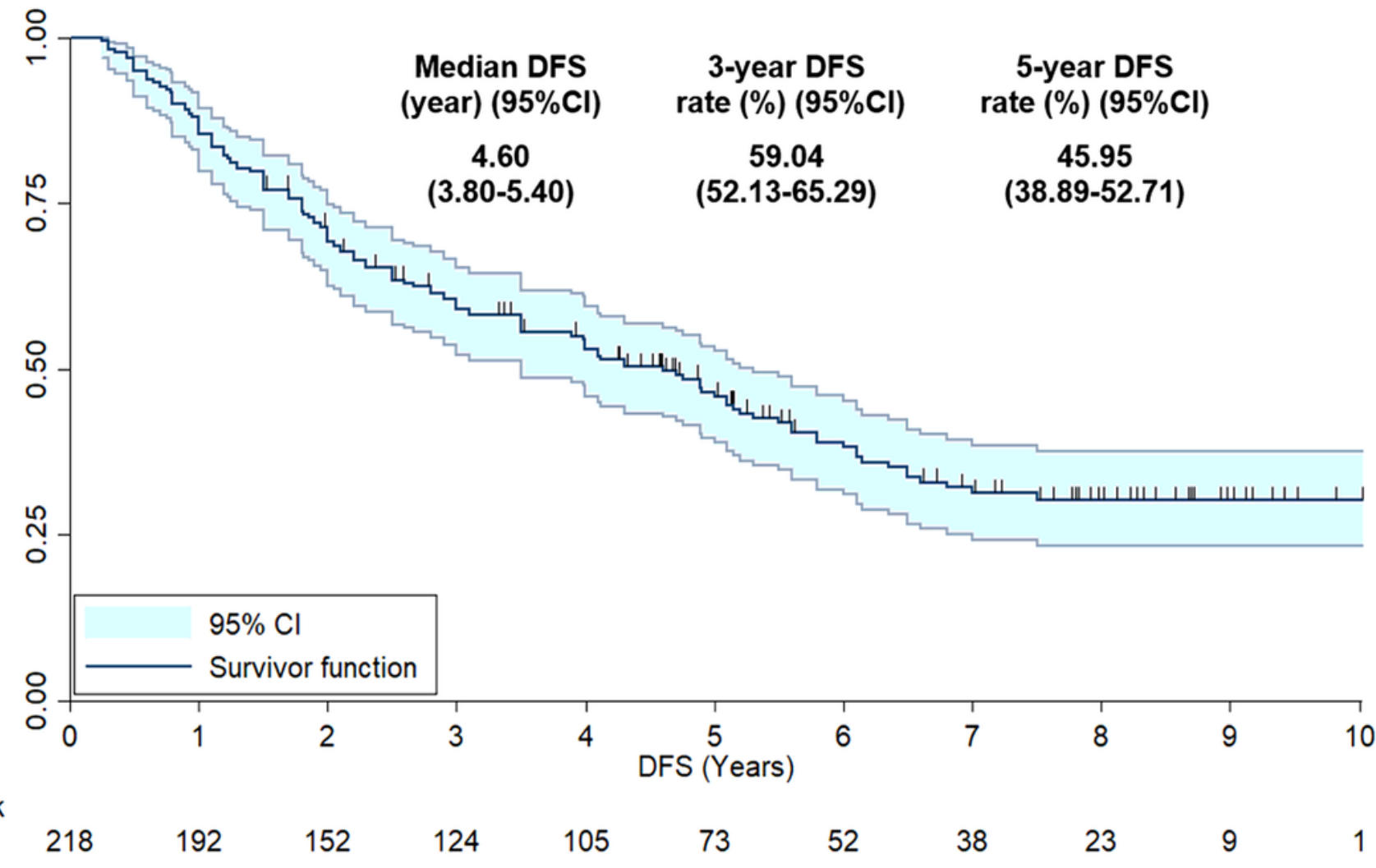

Figure 2

Disease free survival of the 218 patients with colorectal cancer receiving capecitabine-based adjuvant chemotherapy 


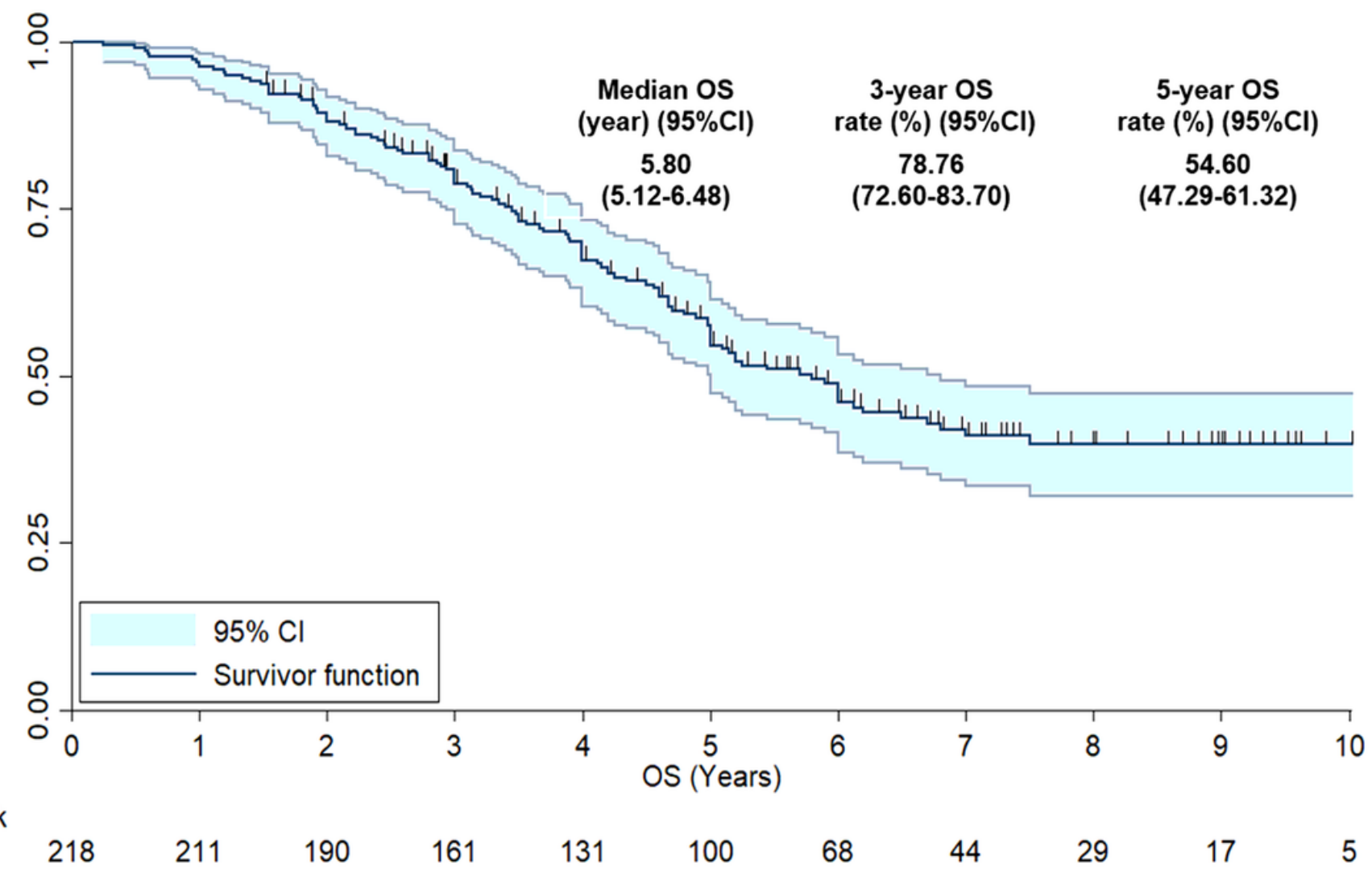

Figure 3

Overall survival of the 218 patients with colorectal cancer receiving capecitabine-based adjuvant chemotherapy

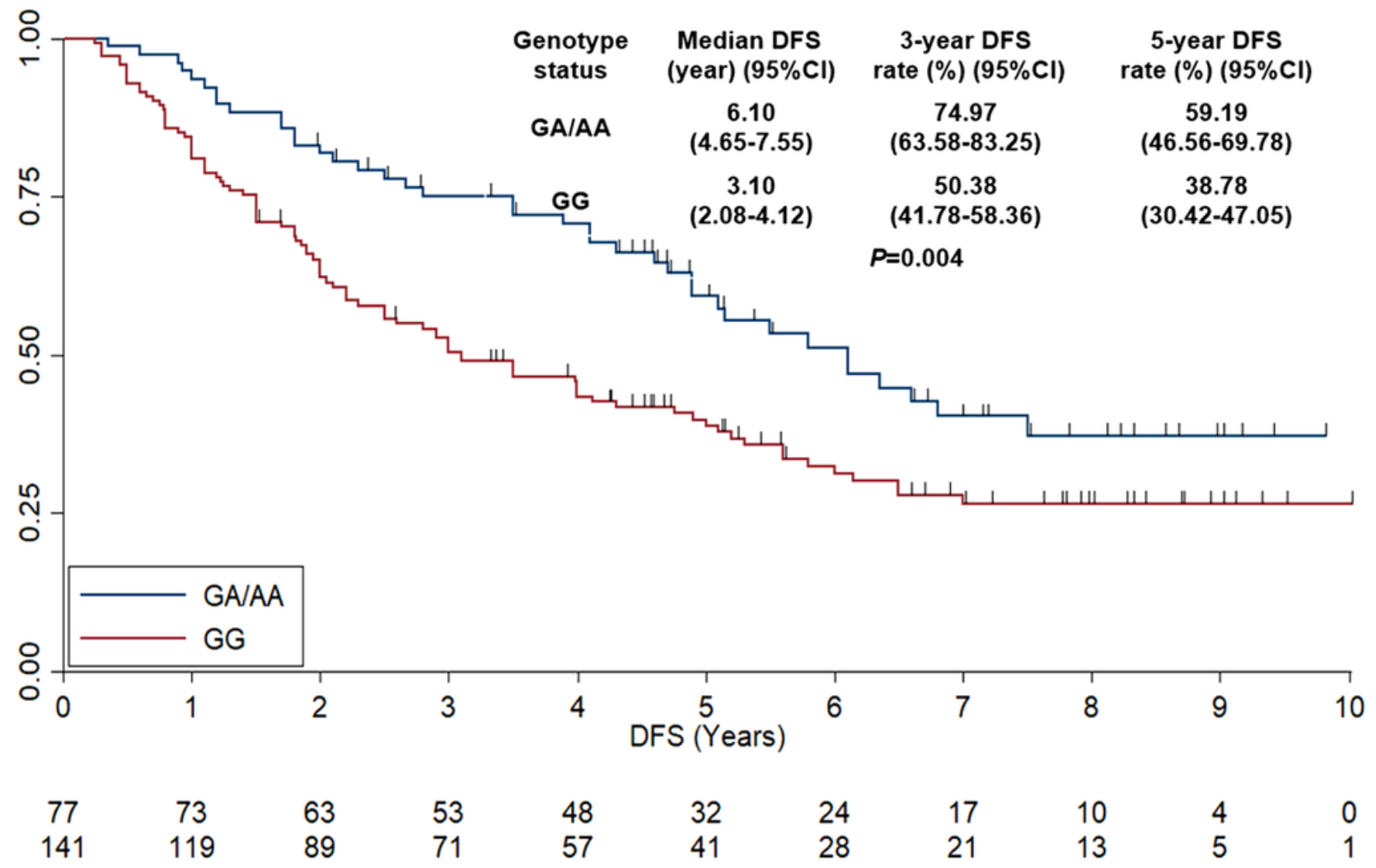




\section{Figure 4}

Disease free survival of the 218 patients with colorectal cancer according to TYMP rs11479 genotype status

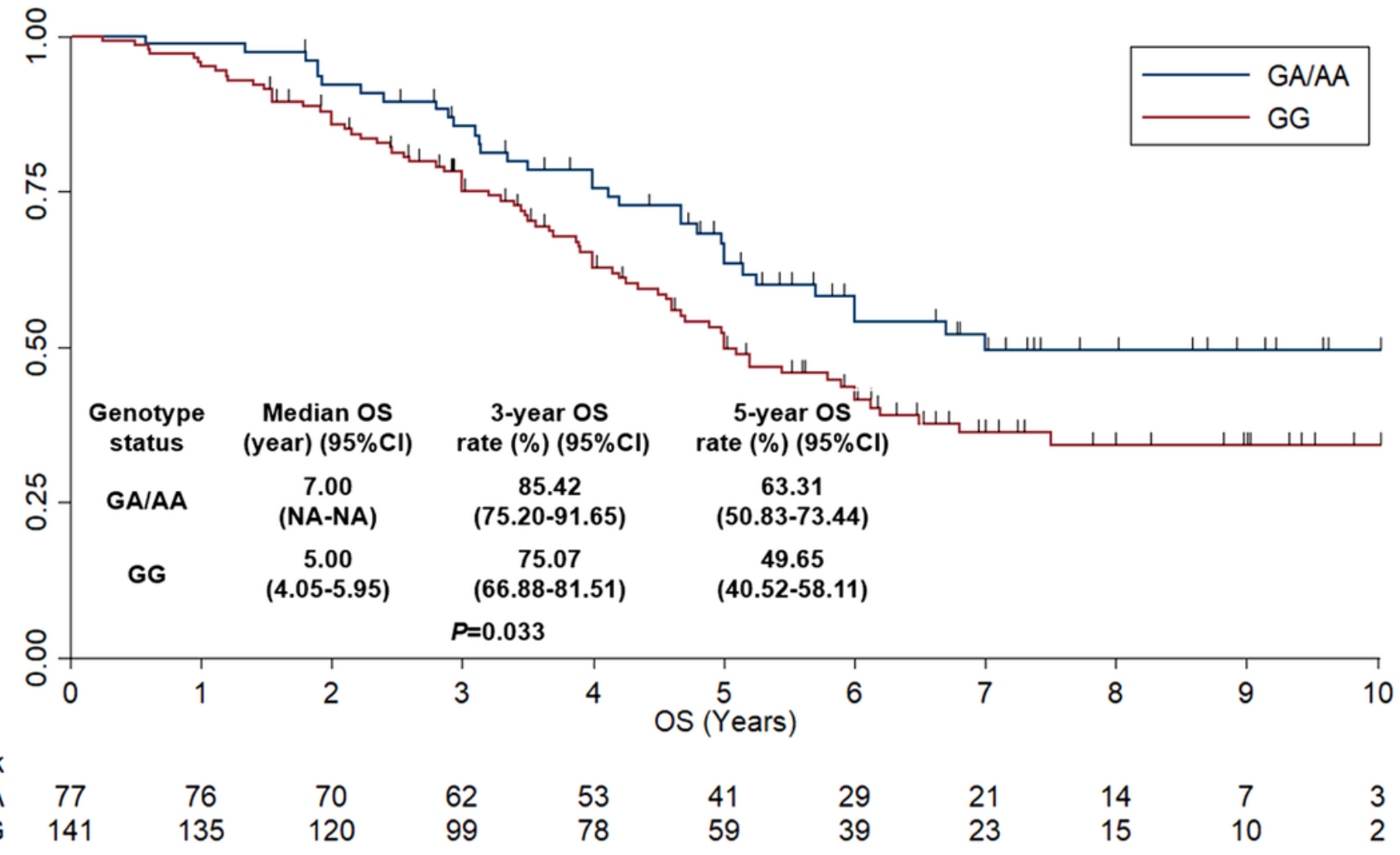

\section{Figure 5}

Overall survival of the 218 patients with colorectal cancer according to TYMP rs 11479 genotype status 


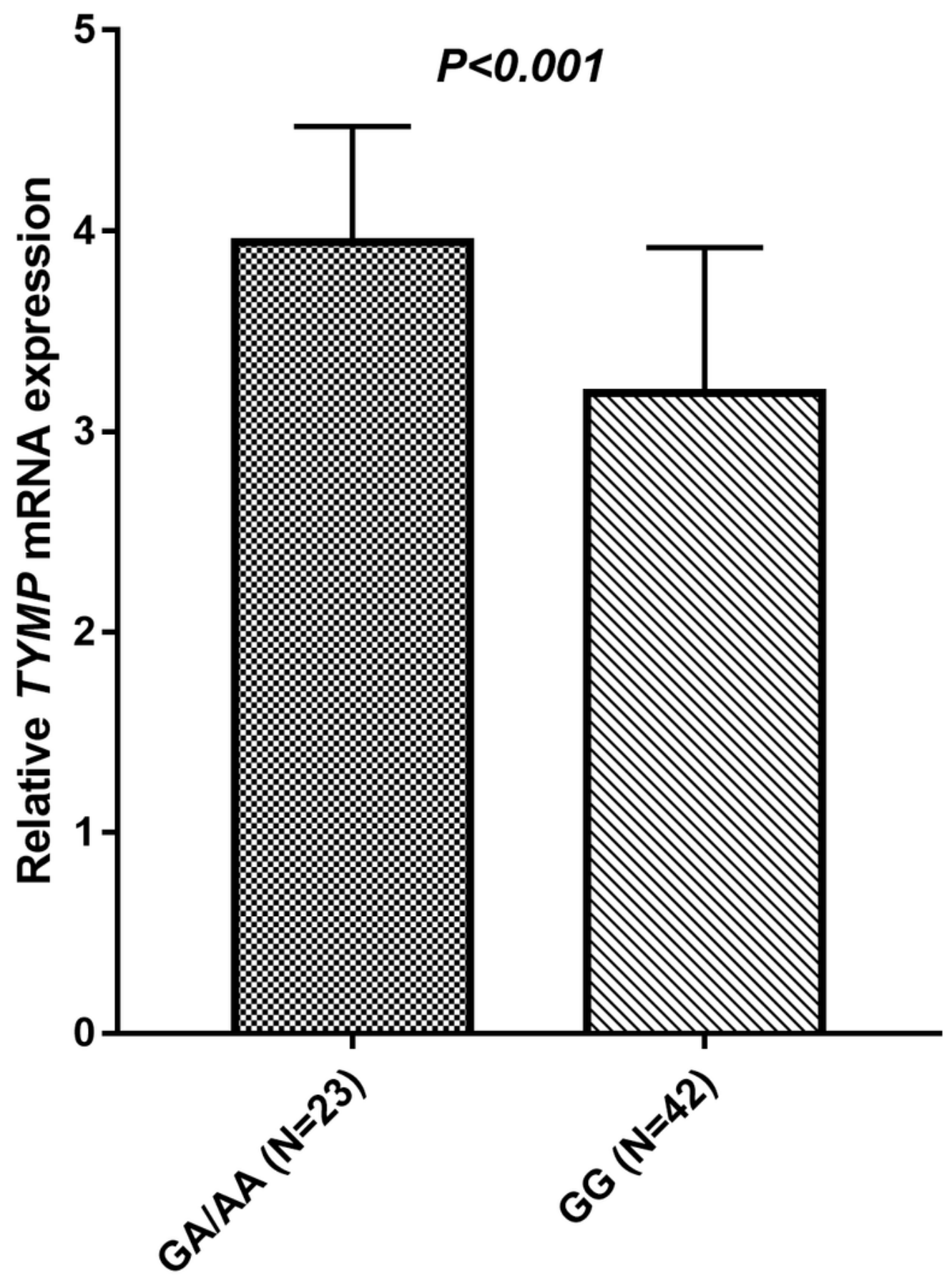

Figure 6

Relative expression level of TYMP mRNA according to TYMP rs11479 genotype status 